\title{
Prevalencia de Artrtitis Reumatoide en dos poblaciones originarias de Argentina. Estudio de base comunitaria: ¿“Dos caras de una misma moneda"?
}

\author{
Prevalence of Rheumatoid Arthritis in two indigenous \\ communities from Argentina. A community based study: \\ "Two sides of the same coin"?
}

\begin{abstract}
Rosana Quintana ${ }^{1,2}$; Vicente Juárez ${ }^{1,3}$; Adriana Silvestre ${ }^{1}$; Mariana Aciar ${ }^{1,3}$; Romina Nieto ${ }^{1,2}$; María E. Crespo ${ }^{1,3}$; Mario Goñi ${ }^{1}$; Natalia Estrella ${ }^{1,3}$; Emilio Buschiazzo ${ }^{1,3}$; Nora Mathern ${ }^{1 ;}$ Natalia Cucchiaro ${ }^{1,3}$; Ingris Pelaez-Ballestas ${ }^{1,4}$ y Bernardo Pons- Estel ${ }^{1,5}$.

${ }^{1}$ Grupo Latinoamericano de Estudios de Enfermedades Reumáticas en Pueblos Originarios (GLADERPO). ${ }^{2}$ Facultad de Ciencias Médicas. Universidad Nacional de Rosario. Rosario; Argentina.

${ }^{3}$ Hospital Señor del Milagro. Salta; Argentina.

${ }^{4}$ Hospital General de México “Dr. Eduardo Liceaga”. Ciudad de México; México.

${ }^{5}$ Centro Regional de Enfermedades Autoinmunes y Reumáticas (GO-CREAR). Rosario; Argentina.
\end{abstract}

Autor por correspondencia: Rosana Quintana - rosanaquintana@gmail.com Conflicto de intereses: no presenta.

\begin{abstract}
Resumen
La artritis reumatoide (AR) es una patología crónica que genera limitación funcional, impactando en la calidad de vida de los pacientes y de su entorno familiar. El objetivo de este estudio fue estimar la prevalencia de AR en las comunidades de pueblos originarios qom y wichi de la ciudad de Rosario y Misión Chaqueña (Salta); además de comparar las características entre ambas. Se realizó un estudio transversal, epidemiológico, de base comunitaria, utilizando la metodología Community Oriented Program for the Control of Rheumatic Diseases (COPCORD). Los individuos con dolor músculoesquelético (casos positivos) fueron evaluados en forma consecutiva por médicos generales y referenciados dentro de la semana al reumatólogo para diagnóstico y eventual tratamiento. La prevalencia de AR fue de 3\% (qom) y 3.2\% (wichi). Las características de la AR fueron similares en ambas comunidades en relación a la actividad medida por Disease Activity Score-28 (DAS 28) ( $p$ 0,341 ) pero no en la limitación funcional medida por Health Assessment Questionnaire Disability Index (HAQ) (p 0,031). Existió un retraso promedio en el diagnóstico de 2 años. El 45\% y 66\% (p 0,100) de los pacientes qom y wichi tuvieron cambios radiográficos propios de una enfermedad avanzada. Más del 90\% fueron seropositivos para factor reumatoideo y anticuerpos antipéptido citrulinado cíclico. El 42\% y 29\% (p 0,246) de los pacientes qom y wichi tenían antecedentes familiares de AR. Las prevalencias de AR en ambas comunidades fueron altas, con marcada agresividad de la enfermedad, así como impactante limitación funcional. El retraso en el diagnóstico es un factor fundamental para mejorar el pronóstico de esta patología.
\end{abstract}

Palabras claves: Metodología COPCORD. Prevalencia. Artritis reumatoide. Poblaciones originarias. 


\begin{abstract}
Rheumatoid arthritis (RA) is a chronic pathology that generates functional limitation, impacting the quality of life of patients and their families. The objective of this study was to estimate the prevalence of RA in the qom and Wichi indigenous communities of the cities of Rosario and Misión Chaqueña (Salta); in addition to comparing the characteristics between both. A cross-sectional, epidemiological, community—based study was carried out using the Community Oriented Program for the Control of Rheumatic Diseases (COPCORD) methodology. Individuals with musculoskeletal pain (positive cases) were evaluated consecutively by a general physician and referred within a week to the rheumatologist for diagnosis and eventual treatment. The prevalence of RA was 3\% (qom) and 3.2\% (wichi). The characteristics of RA were similar in both communities in relation to activity measured by Disease Activity Score-28 (DAS 28) ( $\mathrm{p}$ 0.341) but not in functional limitation measured by Health Assessment Questionnaire Disability Index (HAQ) (p 0.031). There means delay in diagnosis was 2 years. A 45\% and 66\% (p 0.100) of the qom and wichi patients had typical radiographic changes of advanced disease. More than $90 \%$ were seropositive for rheumatoid factor and cyclic citrullinated peptide antibodies. A $42 \%$ and 29\% (p 0.246) of the qom and wichi patients had a family history of RA. The prevalence of RA in both communities was high, with marked aggressiveness of the disease, as well as functional limitation. The delay in diagnosis is a fundamental factor to improve the prognosis of this pathology.
\end{abstract}

Keywords: COPCORD methodology. Prevalence. Rheumatoid arthritis. Indigenous communities.

\title{
Introducción
}

Losdoloresmúsculo-esqueléticosylasenfermedades reumáticasrepresentanunamplioyheterogéneo grupo de patologías asociadas con discapacidad, deterioro de la calidad de vida y algunas de ellas con una mayor mortalidad. En el 2000 se lanzó oficialmente, en la sede de la Organización Mundial de la Salud (OMS) Ginebra, la Década (2000-2010) de la Articulación y el Hueso (w.w.w.nonejointdecade. org), jerarquizando a las enfermedades músculo-esqueléticas como un problema sanitario de relevancia debido a la discapacidad secundaria y en consecuencia a una mayor utilización de recursos de salud (1).

La OMSy la Liga Internacional de Asociaciones de Reumatología(ILAR), desarrollaron un programa denominado Community Oriented Program for the Control of Rheumatic Diseases (COPCORD), con el objetivo de obtener, de forma sencilla y económica, datos sobre enfermedades reumáticas y músculo-esqueléticas en países en vía de desarrollo. La metodología COPCORD ha sido utilizada en varios países (2-5).

En Latinoamérica (LA), cerca de 10\% de su población global pertenece a pueblos indígenas. Estas poblaciones presentan un perfil de patologías diferentes y en relación a las patologías reumáticas, en particular Artritis Reumatoide (AR), una forma de presentación más agresiva y peor pronóstico (6).

En octubre de 2009 se creó el “Grupo Latino Americano De Estudio de Enfermedades Reumáticas en Pueblos Originarios” (GLADERPO). Su objetivo principal fue en una primera etapa, realizar estudios epidemiológicos, antropológicos y genéticos, para luego, en una segunda etapa, dar lugar a procesos de intervención en la población afectada. Actualmente los estudios se están desarrollando en población indígena de Argentina, México, Ecuador y Venezuela.

En Argentina, existen antecedentes de estudios previos de prevalencia de AR, pero enfocados en la obtención de datos a través de registros hospitalarios y ambulatorios, no en la detección comunitaria $(7,8)$.

Los qom o Tobas, pertenecen al grupo de los “Guaycurúes”. En sus orígenes habitaban una extensa región del norte de Argentina, extendiéndose a países limítrofes como Bolivia y Paraguay, denominada el "Gran Chaco". Con la llegada del "hombre blanco", la apropiación de sus tierras y desforestaciones de los bosques, se vieron forzados a abandonar su hábitat natural y migrar a varias ciudades de Argentina, entre ellas a Rosario (9).

Los wichis o Matacos, pertenecen al grupo de la familia lingüística Mataco- maca (10), se establecieron hace 4.000 a 5.000 años en la región del Gran Chaco (en quechua significa territorio de caza), geográficamente abarcaba zona norte de Argentina, Bolivia, Paraguay y Brasil, siendo un área de diversidad demográfica, lingüística y cultural (10). 
El objetivo de este estudio es estimar la prevalencia de AR en las comunidades de pueblos originarios qom y wichi de la ciudad de Rosario y Misión Chaqueña “El Algarrobal” (Salta), así como comparar las características de la AR entre las dos comunidades.

\section{Materiales y métodos}

Se realizó un estudio epidemiológico, de corte transversal analítico y base comunitaria, en la población qom de la ciudad de Rosario y en la comunidad wichi de Misión Chaqueña "El Algarrobal”, en la provincia de Salta, Argentina.

\section{Población de Estudio}

Se incluyeron individuos $\geq$ de 18 años, autoreconocidos como qom y wichi, residentes por un periodo mayor a 6 meses en la ciudad de Rosario y en la comunidad de Misión Chaqueña “El Algarrobal”, respectivamente.

La ciudad de Rosario está ubicada a 306 km al norte de la capital del país, con una población de más de 1.190.000 habitantes (11). Los qom se ubican en tres barrios, en los distritos norte (barrio Travesía), noroeste (barrio Pumitas) y oeste (barrio Rouillón), el de mayor densidad poblacional.

La comunidad de Misión Chaqueña "El Algarrobal”, se encuentra ubicada geográficamente en el departamento General San Martin, en la provincia de Salta, a una distancia de 1729 km al Norte de la ciudad de Buenos Aires. Su acceso es a través de $40 \mathrm{~km}$ de ruta no pavimentada, desde la ciudad más cercana, Embarcación. Carece de un sistema de urbanización.

Debido a la falta de datos concretos sobre la cantidad de individuos residentes, fue necesario implementar un censo como parte de la estrategia metodológica en ambas poblaciones. La realización del censo fue en forma concomitante con la implementación de la encuesta, a través de la visita al domicilio por parte del equipo de trabajo; documentando todos los individuos $\geq$ de 18 años, autoreconocidos como qom y wichi que vivían en forma estable ( $\geq 6$ meses) en esa vivienda.

\section{Cuestionario}

Como instrumento de recolección de datos se utilizó el cuestionario COPCORD, el cual fue previamente adaptado transculturalmente y validado por el grupo GLADERPO (12). La aplicación del cuestionario estuvo a cargo de encuestadores (estudiantes de medicina, enfermeros y agentes sanitarios), previamente entrenados, en compañía de coordinadoras de terreno, médicos y representantes de la comunidad que actuaban como facilitadores - traductores bilingües. El cuestionario COPCORD consta de diferentes secciones: una sección explicativa, que incluye, datos de filiación, historia laboral y comorbilidades auto reportadas, una sección para identificar el dolor músculo— esquelético en los últimos siete días o alguna vez en su vida, incluyendo características tales como gravedad, limitación y adaptación, así como búsqueda de atención y tratamientos médicos y/o tradicionales y finalmente una sección destinada a evaluar la capacidad funcional, medida a través del instrumento Health Assessment Questionnarie disability index (HAQ-DI) corto y validado (13), el cual es autoadministrado y mide la capacidad funcional del individuo en la última semana.

Se elaboró además un cuestionario con indicadores socioeconómicos y sanitarios incluyendo tiempo de residencia, grado de instrucción formal, cobertura de salud y características de la vivienda.

\section{Trabajo de campo}

Como punto inicial, se llevó a cabo una prueba piloto en ambas comunidades, con el objetivo de realizar la adaptación cultural y la validación del cuestionario.

El equipo de trabajo en terreno incluyó: coordinadores, encuestadores, médicos clínicos y reumatólogos, acompañados de individuos referentes de las dos comunidades. La implementación de la encuesta se realizó a través de visitas domiciliarias puerta a puerta. En caso de que el individuo no se encontrara en su domicilio, se repetían las visitas hasta un total de cinco veces, en diferentes horarios y días de la semana, para catalogarlo como ausente. Se comenzaba la visita domiciliaria con la presentación del facilitador - traductor explicando el objetivo del estudio, la confidencialidad y la futura utilización de los datos obtenidos. Se invitaba a participar y se le entregaba el consentimiento informado, dándole un espacio de tiempo para su lectura y comprensión. En caso de que el individuo fuera analfabeto, se explicaba verbalmente el procedimiento. La firma del consentimiento informado se realizaba junto a un testigo, el traductor- facilitador y el encuestador. 
Realizada la encuesta, y en caso de que la misma resultara positiva (presencia de dolor músculoesquelético en la última semana o en algún momento de la vida), el individuo era evaluado por un médico en terreno, entrenado en el reconocimiento de enfermedades reumáticas y, ante la sospecha clínica de una enfermedad reumática, se realizaba la derivación a un médico reumatólogo dentro de la semana, para el diagnóstico y eventual tratamiento. En caso de poliartritis de grandes y pequeñas articulaciones, se realizaron en todos los individuos: dosaje FR [(factor reumatoideo), a través del método de turbidimetría; valor de referencia $\geq 15 \mathrm{IU} / \mathrm{ml}]$, Anti- CCP [(anticuerpos antipéptido citrulinado cíclico), a través del método de quimioluminiscencia, valor de referencia $\geq 5,0 \mathrm{U} / \mathrm{ml}]$ y velocidad de eritro sedimentación (VES) medida en milímetros; así como radiografías de manos y pies. El reumatólogo realizó el diagnóstico según criterio estandarizados para AR (14).

En relación a la AR, se diseñó una historia clínica unificada, donde se evaluaron las siguientes variables: retraso en el diagnóstico definida como el tiempo transcurrido entre el comienzo de los síntomas y el diagnóstico de la AR; antecedentes familiares de AR; seropositividad definida como positividad del FR y Anti-CCP; actividad de la enfermedad medida a través del score DAS 28 VES (Disease Activity Score); a través del cálculo matemático que incluye el recuento total de articulaciones dolorosas e inflamadas, el dosaje de VES y la valoración global del paciente de la enfermedad a través de una escala visual análoga; se categoriza según su valor: DAS28 <2.6 en remisión clínica, DAS28 > =2.6y $<=3.2$ en actividad baja de la AR, DAS28 $>3.2 \mathrm{y}<=5.1$ en actividad moderada y DAS28 $>5$, 1 en actividad alta de la AR; (15); funcionalidad medida a través del score HAQ-DI (Health Assessment Questionnaire disability index) (13); presencia de erosiones en radiografías de manos y/o pies y comorbilidades más frecuentes. La lectura de las radiografías estuvo a cargo del mismo médico reumatólogo con mayor experiencia clínica y se categorizaron como ausencia o presencia de erosiones.

\section{Aspectos éticos}

En la ciudad de Rosario, el estudio fue aprobado para su implementación el 22 de diciembre de 2010 por el Comité de Ética en Investigación de la Secretaría de Salud de la Municipalidad de Rosario (resolución número 1659/2009), con el aval del Ministerio de Salud (resoluciones 1619/2010 y 0127/2011) y del Ministerio de Desarrollo Social de la provincia de Santa Fe, la Asociación de Reumatología de la Provincia de Santa Fe, la Facultad de Ciencias Médicas de la Universidad Nacional de Rosario y los representantes de las organizaciones qom de la ciudad. El estudio fue registrado (orden de registro número 13) en el Comité Provincial de Bioética de la Provincia de Santa Fe, el 5 de Julio de 2012.

En la ciudad de Salta, el estudio fue aprobado por el comité de Ética del Hospital Señor del Milagro y el Ministerio de Salud Pública de la Provincia de Salta (expediente $N^{\circ} 0100134-6738 / 2017-0$ ) y avalado por la Sociedad Argentina de Reumatología, la Sociedad Salteño- Jujeño de Reumatología y el Ministerio de Asuntos Indígenas y Desarrollo Social de la Provincia de Salta.

\section{Análisis Estadístico}

Se realizó un análisis exploratorio de las variables incluidas en el modelo teórico, reportando medidas de tendencia central y de dispersión en variables continuas y frecuencias absolutas y relativas en variables ordinales nominales o categóricas y un análisis comparativo de variables con un nivel de significancia de 0,05.

Se realizó un análisis bivariado de cada una de las variables de estudio, utilizando análisis de varianza (ANOVA) de una y dos vías para las variables continuas y Chi cuadrado para las variables ordinales, nominales y categóricas. El HAQ-DI fue categorizado según el valor, con un punto de corte de 0,8 (13). Este punto de corte fue estudiado previamente por investigadores del grupo GLADERPO como predictor de limitación funcional (13). El denominador utilizado en ambas poblaciones fue el resultado del censo poblacional realizado durante el desarrollo del trabajo de campo.

El análisis estadístico se realizó con el paquete estadístico SPSS (Statistics Standard Edition 22).

\section{Resultados}

La población censada perteneciente a la comunidad qom residente en forma estable en los tres barrios de la ciudad de Rosario, $\geq$ de 18 años, fue de 2.157 individuos. De estos, 1.759 (81,6\%), participaron en el estudio (103 en la prueba piloto y 1.656 en el estudio extendido). Se consideraron ausentes 322 (14,9\%) individuos y $76(3,5 \%)$ no aceptaron participar. 
La población censada $\geq 18$ años de edad perteneciente a la comunidad wichi fue de 717 individuos, 648 participaron en el estudio, representando el 90,4\% de la población. Estuvieron ausentes 41(5,7\%) y no aceptaron participar 28 (3,9\%).

El 53,7\% y el 48,5\% de los encuestados reportaron dolor músculo-esquelético en los últimos siete días y/o alguna vez en la vida, en la comunidad qom y wichi, respectivamente.

Luego de la evaluación reumatológica se diagnosticaron 40/1.656 (2,4\%) individuos con AR en la comunidad qom, luego de la primera etapa del proyecto, que se continuó con la creación de una cohorte comunitaria, dondese agregaron 25 pacientes más, llevando la prevalencia a3\%(65/2.157). En la comunidad wichi se detectaron $21(3,2 \%)$ pacientes con AR.

Las características generales y las comparaciones entre ambas comunidades se describen en la tabla 1. Las variables con significancia estadísticas fueron: mayor diagnóstico previo en la comunidad qom (33,3\% versus 61,5\%; p 0,024) y mayor limitación funcional en la comunidad qom medida por HAQ-DI (76,9\% versus 52,3\%; p 0,031). Así mismo la comunidad wichi presentó una demora en el diagnóstico significativamente superior a la de la comunidad qom (mediana de 25,5 meses versus 12,0; p 0,024). Las comorbilidades más frecuentes en ambas comunidades fueron: hipertensión, diabetes, enfermedad de Chagas y tuberculosis.

Tabla 1. Características generales y comparación de los pacientes diagnosticados de AR de la comunidad wichi y qom

\begin{tabular}{|l|l|l|l|}
\hline Variable & wichi & qom & $\mathbf{p}$ \\
\hline Mujeres, n (\%) & $\mathbf{n = 2 1}$ & $\mathbf{n = 6 5}$ & \\
\hline Edad, años, media (DE) & $19(90,4)$ & $58(89,2)$ & 0,999 \\
\hline Diagnóstico previo, n (\%) & $44,09(7,1)$ & $39,57(12,4)^{(* 7)}$ & 0,050 \\
\hline Antecedentes familiares de AR,n (\%) & $7(33,3)$ & $40(61,5)$ & 0,024 \\
\hline Seropositividad ${ }^{\dagger}, \mathrm{n}(\%)$ & $9(42,8)$ & $19(29,2)$ & 0,246 \\
\hline Presencia de erosiones, n (\%) & $16(76,1)$ & $58(89,2)$ & 0,155 \\
\hline DAS 28 VES, mediana (RIQ) & $14(66,6)$ & $28(45,9)^{\left(*_{4}\right)}$ & 0,100 \\
\hline HAQ-DI $\geq 0,8, \mathrm{n}(\%)$ & $5,56(2,5)^{(* 8)}$ & $4,74(1,3)^{\left(*^{*}\right)}$ & 0,341 \\
\hline Demora en el diagnóstico (meses), mediana (RIQ) & $11(52,3)$ & $50(76,9)$ & 0,031 \\
\hline
\end{tabular}

Abreviaturas: DE(Desvío estándar); RIQ(rango interquartil); ${ }^{\dagger}$ Positividad de FR (factor reumatoideo) o/y Anti- CCP (anticuerpos antipéptido citrulinado cíclico); DAS 28 VES (Disease Activity Score); HAQ-DI (Health Assessment Questionnaire disability index); "tiempo desde el comienzo de los síntomas hasta el diagnóstico de AR $;^{(*)}$ datos faltantes.

En la tabla 2, se describe la diferencia entre ambas comunidades en relación al sexo y edad. Observamos la baja frecuencia del sexo masculino en ambas comunidades. La media de edad fue mayor en la comunidad qom en relación a la comunidad wichi. En la Figura 1, se detalla la distribución del sexo y edad en relación a cada comunidad, la proporción en relación al sexo es similar en ambas comunidades, siendo mayoritario el sexo femenino. 
Tabla 2. Distribución de la edad de los pacientes diagnosticados de AR según sexo y comunidad

\begin{tabular}{|l|l|l|l|l|l|l|l|l|l|}
\hline \multirow{3}{*}{ Sexo } & \multicolumn{1}{|l|}{ Wichi } & \multicolumn{1}{|c|}{ Qom } \\
\cline { 2 - 12 } & $\mathrm{n}$ & Media & DE & $\mathrm{N}$ & Media & DE & $\mathrm{n}$ & Media & DE \\
\hline Femenino & 19 & 44,09 & 7,1 & 58 & 39,57 & 12,4 & 77 & 40,25 & 11,5 \\
\hline Masculino & 2 & 42,50 & 14,8 & 7 & 46,33 & 10,1 & 9 & 45,38 & 10,4 \\
\hline Total & 21 & 44,10 & 7,1 & 65 & 39,57 & 12,4 & 86 & 40,77 & 11,4 \\
\hline
\end{tabular}

Abreviaturas: DE (Desvío estándar)

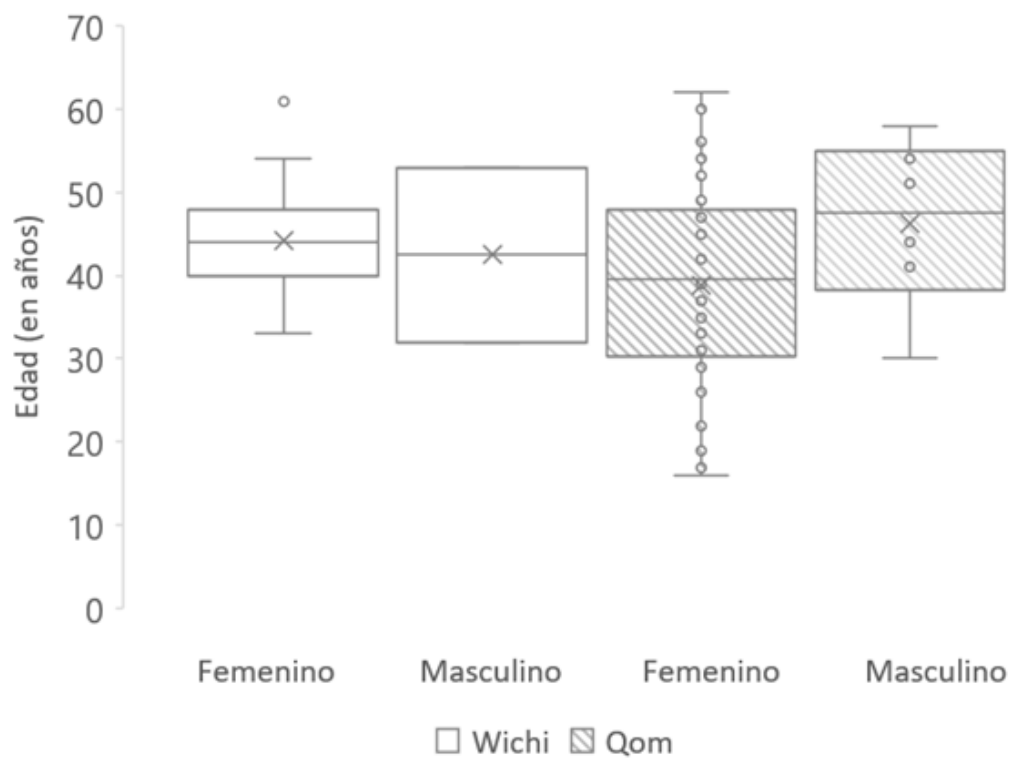

Figura 1: Distribución de la edad pacientes diagnosticados de AR según sexo y comunidad.

\section{Discusión}

Debe destacarse que estas dos comunidades son las primeras en Argentina, donde el grupo GLADERPO ha implementado la metodología COPOCORD. Uno de los hallazgos más relevantes fue la alta prevalencia de la AR en ambas comunidades. Siendo las más altas reportadas en LA aplicando la misma metodología $(16,17)$. Igualmente se han descrito prevalencias mayores en algunos pueblos nativos de Norteamérica, alcanzando hasta un 5-7\%, siendo la comunidad Chippewa $(6,8-7,1 \%)$, Pima (5,3\%), Yakima $(3,4 \%$ en mujeres) y Tingit (2,4\%), las más representativas (18). Además, en estas comunidades la forma de presentación es más agresiva y seropositiva, como en nuestro caso. Un estudio realizado en una comunidad canadiense (Manitobans) también reportó una alta prevalencia de AR, comparada con la población caucásica de la misma región, donde se observó que casi el doble de los registros de atención era por parte de la comunidad indígena (40,9\% vs 25,9\%, p 0.001)(19). En Argentina dos estudios describen la prevalencia de AR en población general. Uno de ellos fue realizado en la capital de la provincia de Tucumán, la información se obtuvo a través de registros hospitalarios y ambulatorios, tanto privado como públicos. Se describió una tasa de prevalencia global de AR de 1.97/1000 habitantes (0.2\%) y el 10\% de la población pertenecía a una comunidad indígena (7). El otro estudio fue realizado en la ciudad de Luján, en la provincia de Buenos Aires. Los datos fueron aportados por reumatólogos locales y a través de una encuesta telefónica. La prevalencia fue de $0.9 \%$ y no describen la etnia de los pacientes (8).

Otro hallazgo relevante fue el alto porcentaje, sobre todo en la comunidad qom de familiares con AR. Esto no ha sido reportado en ningún otro estudio COPCORD. En comunidades de pueblos indígenas de Norteamérica, se ha observado que la posibilidad de padecer AR entre familiares de primer grado, es muy alta, incluso de tener serologías positivas, sin desarrollar la enfermedad (20,21). 
Otro punto a destacar es que ambas poblaciones presentaron alto porcentaje de seropositividad para FR y CCP, un alto grado de actividad de la enfermedad medida por DAS 28 y una marcada limitación funcional medida por HAQ-DI. En este sentido, Nagaraj y col. (22) compararon la forma de presentación y la evolución en una cohorte de artritis temprana canadiense. Las características basales de pacientes caucásicas y pertenecientes a una comunidad originaria fueron similares, pero no la evolución de los mismos, teniendo los pacientes indígenas una peor evolución y respuesta al tratamiento. Los autores remarcan que el problema es más estructural y está relacionado a la inequidad en salud. Marrone (23) describe que el problema de estas poblaciones vulnerables radica en la falta de accesibilidad al sistema de salud y la inequidad en salud. Ubica como las principales barreras a: residencia rural, bajo nivel educacional y socioeconómico, así como la falta de competencia cultural de los profesionales de la salud.

Otro punto a remarcar es el retraso en el diagnóstico en ambas comunidades, siendo significativamente mayor en la comunidad wichi. Pelaez y col (24) publicaron en el 2015 una revisión sobre el proceso de búsqueda de ayuda en el diagnóstico de AR en LA, demostrando que los servicios de salud son inaccesibles, fragmentados y limitados, sobre todo en la atención de pueblos originarios. Lamentablemente el retraso en el diagnóstico es disímil en LA, representando la desigualdad en salud. Las variables asociadas a mayor retraso son: bajo nivel socioeconómico, bajo nivel educacional, residencia rural y pertenencia a grupo étnico minoritario. Esta situación puede ser claramente visibilizada en las dos comunidades estudiadas, no solo el retraso en el diagnóstico, sobre todo relacionado al contexto rural de la comunidad wichi, sino también la magnitud del mismo en ambas comunidades a través de la presencia de erosiones, que refleja la evolutividad de una enfermedad agresiva, así como la limitación funcional descripta en ambas.

Las comorbilidades asociadas a la AR fueron similares en ambas y a otras comunidades de Canadá, Australia y Nueva Zelanda (25,26). Loyola Sanchez y col (27) describen que las comunidades indígenas acceden a los servicios de salud a través de las hospitalizaciones o urgencias y no a través de la consulta programada y especializada.

El grupo GLADERPO (28), publicó un artículo donde analizan las interacciones entre las enfermedades reumáticas, las comorbilidades y factores socioeconómicos, en ocho comunidades originarias de Argentina, México y Venezuela. El análisis fue realizado a través de un modelo sindémico (29), destacando la importancia de factores culturales y étnicos que se congregan, impactando negativamente en la vida de los individuos con alguna enfermedad reumática.

Otro dato a destacar en ambas comunidades fue la alta prevalencia de dolor músculo- esquelético en general, asociado a una importante limitación funcional medida por HAQ-DI, sobre todo en la comunidad qom, pudiendo relacionarse, entre otros factores, a la actividad laboral asociada a la carga y la repetición. En los estudios COPCORD realizados en pueblos indígenas de Guatemala y Australia, la prevalencia de dolor músculo-esquelético fueron menores 4,5\% y 33\% respectivamente. En los estudios COPOCORD realizados en pueblos indígenas de Guatemala (30) y Australia (31), las prevalencias de dolor músculo-esquelético fueron menores, 4,5\% y 33\% respectivamente. El estudio realizado en la zona rural de Irán, describió una prevalencia de dolor del 41.9\%. En relación a los estudios realizados en LA, México reportó una prevalencia del 25,5\% (32), Cuba 43,9\% (33), Venezuela 22,4\% (34), Brasil 30,9\% (35), Perú 46,6\% (36) y Ecuador 32,5\% (37).

Entre las principales limitaciones de este trabajo, podemos enumerar: el bajo número de la muestra; los datos faltantes para un mejor análisis y las diferencias culturales entre la comunidad y el equipo de trabajo.

Como principal fortaleza, este tipo de estudio comunitario ha sido uno de los primeros realizados en Argentina en comunidades originarias, tanto en contexto urbano como rural.

Como conclusión, las prevalencias de AR en ambas comunidades fueron altas, con marcada agresividad de la enfermedad, así como una importante limitación funcional. El retraso en el diagnóstico es un factor fundamental para mejorar el pronóstico de esta patología.

\section{Agradecimientos}

A la Sociedad Argentina de Reumatología; a la Municipalidad de la Ciudad de Rosario; Secretaría de Salud de la Municipalidad de Rosario; Ministerio de Salud de la Provincia de Santa Fe; Facultad de Ciencias Médicas, Universidad Nacional de Rosario; Equipos de Salud de Atención Primaria de la Salud tanto municipales como provinciales de la ciudad de Rosario; Representantes de la comunidad qom de la ciudad de Rosario; Comunidad qom en su totalidad por su generosidad y compromiso en la realización del proyecto; Grupo de CEAPROS liderado por la Antropóloga 
Marcela Valdata perteneciente a la Facultad de Humanidades y Artes, Universidad Nacional de Rosario; Sociedad Salteña Jujeña de Reumatología; Ministerio de Salud de la Provincia de Salta; Profesionales de la salud de la ciudad de Embarcación; Misión Chaqueña y Ciudad de Salta. A la comunidad wichi por su compromiso.

Fuente de financiamiento: no presenta.

\section{Referencias bibiográficas}

1. Brooks PM (2006) The burden of musculoskeletal disease - a global perspective. Clin Rheumatol 25: 778-81

Darmawan J (2007) Recommendations from the Community Oriented Program for Control of Rheumatic Disease for data collection for the measurement and monitoring of health in developing countries. Clin Rheumatol 26:853-7 Muirden KD (2005) Community Oriented Program for the Control of Rheumatic Diseases: studies of rheumatic diseases in the developing world. Curr Opin Rheumatol 17:153-6 Montenegro RA, Stephens C (2006) Indigenous health in Latin America and the Caribbean. Lancet 3;367(9525):1859—69 Spindler A, Bellomio V, Berman A, Lucero E, Baigorria M, Paz S, et al (2002) Prevalence of rheumatoid arthritis in Tucumán, Argentina. J Rheumatol 29:1166-70 Scublinsky D, Venarotti H, Citera G, Messina OD, Scheines E, Rillo O, et al (2010) The prevalence of rheumatoid arthritis in Argentina: a capture - recapture study in a city of Buenos Aires province. J Clin Rheumatol 16:317-21 Bou Luís C (2003) Los indios Tobas en Rosario, Argentina. http://argentina.indymedia.org/news/2003/04/101871.php C. Messineo and P. Cúneo, "Ethnobiological Classification in Two Indigenous Languages of the Gran Chaco Region: Toba (Guaycuruan) and Maká (Mataco-Mataguayan),” Anthropological Linguistics, vol. 53. Trustees of Indiana UniversityAnthropological Linguistics, pp. 132-169, 2011 Censo 2010. Argentina. http://www.censo2010.indec.gov.ar/index_cuadros.asp Peláez- Ballestas I, Granados Y, Silvestre A, Alvarez- Nemegyei J, Valls E, Quintana R, et al (2014) Culture-sensitive adaptation and validation of the Community-Oriented Program for the Control of Rheumatic Diseases methodology for rheumatic disease in Latin American indigenous populations. Rheumatol Int 34(9):1299-309. doi: 10.1007/s00296—014-2997—z Goycochea-Robles MV, Sanin LH, Moreno-Montoya J, Alvarez-Nemegyei J,Burgos—Vargas R et al (2011) Validaty of the COPCORD core questionnaire as a classification tool for rheumatic diseases J Rheumatol Suppl ;86:31—5 Arnett FC, Edworthy SM, Bloch DA, McShane DJ, Fries JF, Cooper NS et al (1988) The American Rheumatism Association 1987 revised criteria for the classification of rheumatoid arthritis. Arthritis Rheum 31:315-24 Piet L C M van Riel, Lisanne Renskers (2016) The Disease Activity Score (DAS) and the Disease Activity Score using 28 joint counts (DAS28) in the management of rheumatoid arthritis Clin Exp Rheumatol 34(5 Suppl 101):S40—S44. Y. Granados, L. Cedeño, C. Rosillo, S. Berbin, M. Azocar, M. E. Molina, O, et al (2015) Prevalence of musculoskeletal disorders and rheumatic diseases in an urban community in Monagas State, Venezuela: a COPCORD study. Clin. Rheumatol., vol. 34, no. 5, pp. 871-7 S. Guevara-Pacheco, A. Feicán-Alvarado, L. H. Sanín, J. Vintimilla-Ugalde, F. Vintimilla—Moscoso, J, et al (2016) Prevalence of musculoskeletal disorders and rheumatic diseases in Cuenca, Ecuador: a WHO-ILAR COPCORD study. Rheumatol. Int., vol. 36, no. 9, pp. 1195-1204

E. D. Ferucci, D. W. Templin, and A. P. Lanier (2005) Rheumatoid arthritis in American Indians and Alaska Natives: a review of the literature. Semin. Arthritis Rheum., vol. 34, no. 4, pp. 662-7 C. Barnabe, B. Elias, J. Bartlett, L. Roos, and C. Peschken (2008) Arthritis in Aboriginal Manitobans: evidence for a high burden of disease. J. Rheumatol., vol. 35, no. 6, pp. 1145-50

20. Molokhia M, McKeigue P (2000) Risk for rheumatic disease in relation to ethnicity and admixture. Arthritis Res 2:115-125 Marrone S (2007) Understanding barriers to health care: a review of disparities in health care services among indigenous populations. Int J Circumpolar Health Jun 66(3):188-198 Pelaez I, Infante C, Quintana R (2015) Help—seeking trajectory in patients with rheumatoid arthritis. Clin Rheumatol Mar 34(Suppl 1):S17-S28 Canada, the USA, Australia and New Zealand. Rheumatol Int Apr 37(4): 503-521

Poole JL, Cordova JS, Sibbitt WL Jr, Skipper B (2010) Quality of life in American Indian women with arthritis or diabetes. Am J Occup Ther 64(3):496-505 
Zealand, and the United States: a systematic review. Semin Arthritis Rheum Apr 46(5):665-674 of the rheumatic diseases on indigenous people: an invisible syndemic public health problem. Ann Rheum Dis ;77(10):1397-1404. doi: 10.1136/annrheumdis-2018-213625. Epub 2018 Jul 14. Singer M, Bulled N, Ostrach B, et al (2017) Syndemics and the biosocial conception of health. The Lancet; 389:941-50. Obregon-Ponce A, Iraheta I, Garcia-Ferrer H, Bayardo Mejia, Garcia-Kutzbach A (2012) Prevalence of Musculoskeletal Diseases in Guatemala, Central America: The COPCORD Study of 2 Population. J Clin Rheumatol 18(4):170—4. doi: 10.1097/RHU.0b013e3182583803 Minaur N, Sawyers S, Parker J, Darmawan J (2004) Rheumatic disease in an Australian Aboriginal community in North Queensland, Australia. A WHO-ILAR COPCORD Survey. J Rheumatol 31:965-72 Peláez-Ballestas I, Sanin LH, Moreno-Montoya J, Alvarez-Nemegyei J, Burgos—Vargas R, Garza—Elizondo M et al (2011) Epidemiology of the rheumatic diseases in Mexico. A study of 5 regions based on the COPCORD methodology. J Rheumatol Suppl 86:3-8. doi: 10.3899/ jrheum.100951 Reyes-Llerena GA, Guibert-Toledano M, Penedo-Coello A, Perez-Rodriguez A, Baez-Duenas RM, Charchinaro-Vidal R et al (2009) Community - based study to estimate prevalence and burden of illness of rheumatic diseases in Cuba: a COPCORD study. J Clin Rheumatol 15:51-5. doi: 10.1097/RHU.0b013e31819b61cb

34. Granados Y, Cedeño L, Rosillo C, Berbin S, Azocar M, Molina ME et al (2014) Prevalence of musculoskeletal disorders and rheumatic diseases in an urban community in Monagas State, Venezuela: a COPCORD study. Clin Rheumatol Jun 13. [Epub ahead of print] Rodríguez-Senna E, De Barros LP, Silva EO, Costa IF, Pereira LV, Mesquita—Ciconelli R et al (2004) Prevalence of rheumatic diseases in Brazil: a study using the COPCORD approach. J Rheumatol 31:594-7 Gamboa R, Medina M, Acevedo E, Pastor C, Cucho M, Gutierrez C et al (2009) Prevalence of rheumatic diseases and disability in an urban marginal Latin American population. A community based study using the COPCORD model. Rev Peru Reumatol 15:40—6 rheumatic diseases in Cuenca, Ecuador: a WHO-ILAR COPCORD study," Rheumatol. Int., vol. 36, no. 9, pp. 1195-1204, 2016 